EPJ Web of Conferences 66, 05011 (2014)

DOI: $10.1051 /$ epjconf/ 20146605011

(C) Owned by the authors, published by EDP Sciences, 2014

\title{
A novel approach to measure the electric dipole moment of the isotope $129-\mathrm{Xe}$
}

\author{
F. Kuchler ${ }^{1, a}$, P. Fierlinger ${ }^{1}$, and D. Wurm ${ }^{1}$ \\ ${ }^{1}$ Excellence Cluster Universe, Technische Universität München, Boltzmannstr. 2, 85748 Garching, Germany
}

\begin{abstract}
Permanent electric dipole moments (EDM) of fundamental systems are promising systems to find new CP violation beyond the Standard Model. Our EDM experiment is based on hyper-polarized liquid xenon droplets of sub-millimeter size on a micro-fabricated structure, placed in a low-field NMR setup. Implementation of rotating electric fields enables a conceptually new EDM measurement technique, allowing thorough investigation of systematic effects. Still, a Ramsey-type spin precession experiment with static electric field can be realized at similar sensitivity within the same setup. Employing superconducting pick-up coils and highly sensitive LTc-SQUIDs, a large array of independent measurements can be performed simultaneously. With our approach we aim to finally increase the sensitivity on the EDM of ${ }^{129} \mathrm{Xe}$ by more than three orders of magnitude.
\end{abstract}

\section{Introduction}

Despite the success of the Standard Model (SM) over a wide energy scale, it leaves some very fundamental questions unanswered. Observations show no evidence of significant abundances of primordial anti-matter up to the Hubble scale [1]. According to [2], violation of charge-conjugation (C) symmetry and charge- and parity-conjugation (CP) symmetry is one of three required conditions to account for the observed baryon asymmetry in the universe. CP violation (CPV) included in the SM via a phase in the CKM matrix is not sufficient to explain observations, hence new sources of CPV, eg. permanent electric dipole moments (EDM) are searched for. In order to constrain and identify the underlying theory a variety of systems of fundamental particles, atoms and molecules are today's focus of EDM searches [3-6]. Most EDM searches are more and more limited by both systematic effects and statistics, hence new approaches are of significant value to improve current limits.

Here we propose an atomic EDM experiment based on an array of enclosed sub-millimeter droplets of liquid ${ }^{129} \mathrm{Xe}$, with a current experimental limit of $d_{\mathrm{Xe}}=0.7 \pm 3.3 \pm 0.1 \cdot 10^{-27} \mathrm{ecm}$ [4]. Our novel approach allows for an EDM search employing a high-density sample with a volume roughly $10^{4}$ times smaller than previous experiments. The slow self-diffussion in a liquid $(\approx 100 \mu \mathrm{m} / \mathrm{s}) \mathrm{re}-$ duces unwelcome geometric phase effects, which is one of the most significant systematic effects in state-of-the-art EDM searches. It has also been shown that transverse spin-relaxation is slow in liquid xenon and $T_{2}>1000 \mathrm{~s}$ can be achieved [7]. Small sample size allows use of moderate potentials (up to $10 \mathrm{kV}$ ), which, together with small magnetic fields $(1-10 \mu \mathrm{T})$ permits implementation of time-varying electric fields.

\footnotetext{
a e-mail: florian.kuchler@ph.tum.de
} 


\section{Experimental realization}

The ${ }^{129} \mathrm{Xe}$ gas is polarized in a spin-exchange optical pumping (SEOP) [8] flow-through setup and accumulated in a cold trap at liquid nitrogen temperatures in magnetic fields above $20 \mathrm{mT}$. The maximum polarization of 400 mbar of natural xenon (in a $20 \mathrm{~cm}^{3}$ cell) determined by calibration with protons is $\approx 20 \%$ and builds up in about $60 \mathrm{~s}$.

As soon as enough polarized ${ }^{129} \mathrm{Xe}$ is accumulated (ca. 20 minutes) the trap is warmed and ${ }^{129} \mathrm{Xe}$ is transfered to the EDM cryostat. This is performed by cooling the bottom plate of a $20 \mathrm{~mm}$ diameter glass tube inside the EDM setup. This plate of $35 \mathrm{~mm}$ diameter and $2.5 \mathrm{~mm}$ thickness is made of machinable ceramics or quarz and serves as the micro-structured EDM chip, where the actual EDM measurement takes place. The temperature of this micro-fabricated structure can be controlled from $90 \mathrm{~K}$ to $250 \mathrm{~K}$, which enables us to freeze, liquify and evaporate ${ }^{129} \mathrm{Xe}$. The cooling system uses a mixture of warm and cold helium gas which is in thermal contact with the EDM chip. When the glass tube is filled with about $200 \mathrm{~mm}^{3}$ of liquid ${ }^{129} \mathrm{Xe}$ a pressure actuated piston can be used to encapsulate the individual liquid samples on the EDM chip in choosable geometries. For our EDM search cylindrical and (later) spherical shapes will be used, the latter being favored to avoid instabilities, since there is no preferred direction in the liquid volume.

At a distance of $1 \mathrm{~mm}$ below each individual liquid droplet a superconducting pick-up coil made of niobium wire (diameter $2 \mathrm{~mm}$, twelve turns) on sapphire is mounted and thermally connected to 4.2 K. The pick-up coils are tilted at a large angle (eg. $45^{\circ}$ ) to allow detection of magnetic signals transverse to the $B_{0}$ direction (see Fig. 2). A small vacuum gap maintains thermal insulation and a reflection coating of the EDM chip downside reduces heat radiation. Gradiometer pick-up coils are sensitive to the flux change caused by close-by rotating magnetic dipoles $\propto \frac{1}{r^{3}}$, but insensitive to field fluctuations on larger scales. The induced current is measured by connecting the coils to LTc SQUID current sensors (Magnicon). Both the current sensors and the pick-up coils are cooled to $4.2 \mathrm{~K}$ by a closed-cycle cooling machine (Sumitomo). Not using the SQUIDs in a helium bath is technically more difficult, but enables maintenance-free long term operation.

The noise density spectra (see Fig. 1) measured with superconducting gradiometer sensors show the residual magnetic flux noise in the setup and successful operation of the detection system at the intrinsic noise level of the SQUID sensors of $<2 \frac{\mathrm{fT}}{\sqrt{\mathrm{Hz}}}$ at frequencies of 60 to $90 \mathrm{~Hz}$. For an EDM search (either with static or rotating electric fields) the Larmor frequency of ${ }^{129} \mathrm{Xe}$ will preferably be chosen in this low noise region, i.e. $B_{0}=5.1-7.6 \mu \mathrm{T}$.

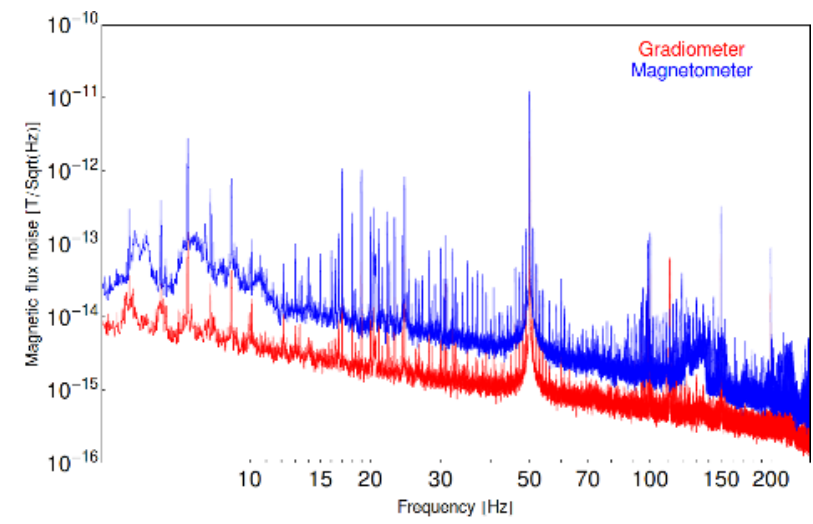

Figure 1. Spectral noise density measured in the magnetically shielded Xenon EDM setup: magnetometer (red) and gradiometer (blue) measurements show mechanical vibrations at low frequencies and electromagnetic noise sources at various frequencies. The overall noise level and most peaks are reduced in the gradiometer spectrum compared to the magnetometer spectrum. Between 60 and 90 $\mathrm{Hz}$ the gradiometer spectrum has a magnetic flux noise at the intrinsic noise level of the SQUID sensors below $2 \frac{\mathrm{fT}}{\sqrt{\mathrm{Hz}}}$

The EDM chip with the ${ }^{129} \mathrm{Xe}$ sample is placed in a low field NMR setup inside a multi-layer $\mu$-metal shield surrounded by a field-compensation system. This allows control of residual gradients 
on a $0.1 \mathrm{nT} / \mathrm{m}$ level resulting in negligible magnetic field deviations over the droplet dimensions of $<50 \mathrm{fT}\left(B_{0} \approx \mu \mathrm{T}\right)$. The small size of the micro-fabricated structure helps generating a very stable and homogeneous constant $B_{0}$ and oscillating $B_{1}$ field over the sample volume. The magnetic fields for all spacial directions are generated by two cosine-theta coils and a cylindrical coil printed on flex-circuits and providing a relative homogeneity of $10^{-4}$ in a cubic volume of $1 \mathrm{~cm}^{3}$. Simulations showed, that this can be improved by a factor of 10 by implementing correction coils.

\section{Xenon EDM search}

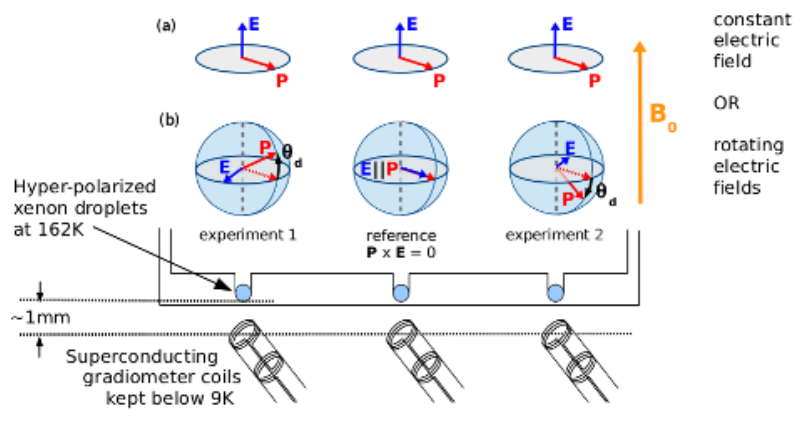

Figure 2. Two options of an EDM search: (a) static electric field applied parallel to the magnetic field, looking for a deviation in precession frequency (b) rotating electric fields $\pm E \perp B_{0}$ with constant phase $\varphi$ (left/right) and zero phase as reference (center) to the precessing polarization vector $P$. Superconducting gradiometer coils detect precession and possible EDM signals.

\section{1 "Conventional" static electric-field measurement}

In this "Ramsey-type" EDM search an electric field $E$ is applied in parallel to a magnetic holding field $B_{0}$. A deviation in Larmor precession frequency for $E=0$ and $E \neq 0$ can be attributed to an electric dipole moment: $\hbar \omega_{L}=\mu B_{0} \pm d E$, with + and - denoting $E$ aligned parallel and anti-parallel to $B_{0}$, respectively. In general, this method of parallel static electric fields strongly relies on highly stable $B_{0}$ fields (or highly accurate knowledge of $B_{0}$ ) to identify and account for false EDM signals.

Two electrodes to apply a static electric field parallel to the magnetic field can be easily implemented by metallizing the downside of the EDM chip and the piston above the liquid ${ }^{129} \mathrm{Xe}$ used for encapsulation (see section 2). By variing the polarity of the applied electric field, a deviation of the spin-precession frequency of ${ }^{129} \mathrm{Xe}$ can be detected.

The sensitivity $\sigma_{\mathrm{dXe}}=\frac{\hbar}{2 E \cdot S N R \cdot T^{3 / 2}}$ of this "Ramsey-type" EDM search, with an applied electric field $E=50 \mathrm{kV} / \mathrm{cm}$, an expected signal-to-noise ratio $S N R=50000$ and an observation time $T=1000 \mathrm{~s}$, will be $\sigma_{\mathrm{dXe}} \approx 10^{-30} \mathrm{ecm}$ in a single measurement.

\subsection{Rotating electric-field measurement}

To overcome the limitations of current EDM measurements we introduce a novel method using a rotating electric field applied perpendicular to the magnetic holding field. Rotating the electric field $E$ with a constant phase to the precessing polarization vector $P$ (see Figure 2) a non-zero EDM leads to a rotation of $P$ according to $\omega_{d}=d E / \hbar$. Note that $\omega_{d}$ is orthogonal to the Larmor precession $\omega_{L}$. An EDM measurement starts by applying a highly accurate oscillating $B_{1}$ pulse to flip the polarization $P$ by an angle $\theta_{1}$, with $P$ initially aligned along $B_{0}$. After a free precession period $\tau$ a second $B_{1}$ pulse flips $P$ by an angle $\theta_{2}=\pi-\theta_{1}$. In case of a finite permanent EDM of ${ }^{129} \mathrm{Xe}$ the polarization is rotated out of the precession plane during free precession, accumulating an angle $\theta_{d}=\omega_{d} \tau$. This 
causes a misalignment of $P$ and $B_{0}$ after the second spin-flip (see Figure 2) resulting in a precession of $P$ around $B_{0}$ at a small tip angle $\theta_{d}=\frac{d E \tau}{\hbar}$.

Applying alternating voltages to eight electrodes embedded around each ${ }^{129} \mathrm{Xe}$ droplet allows individually rotating electric fields, which can be phase-locked to the free precession signal of each droplet. Due to the small size high electric fields of $\pm 50 \mathrm{kV} / \mathrm{cm}$ can be generated with moderate high potentials of $\pm 10 \mathrm{kV}$. In our approach $B_{0}$ fluctuations or drifts do not cause false EDM effects $\left(\omega_{L} \perp \omega_{d}\right)$.

In a three droplet experiment the electric fields can be rotating (i) with a phase $\varphi=\frac{\pi}{2}$, (ii) in phase $\varphi=0$ and (iii) with a phase $\varphi=-\frac{\pi}{2}$ with respect to $P$ (see Figure 2). Note, that precession signals due to a misaligned polarization vector in experiments $(i)$ and (iii) are phase-shifted by $\pi$. Systematic effects caused by rotating electric fields can be accounted for by monitoring the signal detected below a droplet with $E \| P$, since there will be no effect due to an EDM, as $P \times E=0$.

As an example with an electric field of $50 \frac{\mathrm{kV}}{\mathrm{cm}}$ and a free precession period $\tau=1000 \mathrm{~s}$ an EDM of $d=5 \cdot 10^{-30} \mathrm{ecm}$ gives rise to a tip angle $\theta_{d}=\frac{d E \tau}{2 \pi \hbar} \approx 4 \cdot 10^{-7}$. Arbitrarily assuming a droplet diameter of $800 \mu \mathrm{m}$ containing about $4 \cdot 10^{18}$ atoms and a polarization of $20 \%$ the peak-to-peak amplitude difference of experiments with opposite electric field polarity $((i),(i i i))$ is estimated to $170 \mathrm{fT}$. This assumes a pick-up coil distance of $1 \mathrm{~mm}$ and an angle of $45^{\circ}$. At the demonstrated noise level (Figure 1) of the detection system an EDM of $5 \cdot 10^{-30} \mathrm{ecm}$ can be detected in a single measurement at a signal-to-noise ratio of $\approx 15$, when choosing a Larmor frequency in the range $60-90 \mathrm{~Hz}$ and assuming a bandwidth of $30 \mathrm{~Hz}$. Repeating the experiment $10^{4}$ times ( $\approx 150$ days $)$ can improve the statistics by two orders of magnitude. Hence, an EDM sensitivity of order $10^{-32} \mathrm{ecm}$ is the ultimate goal.

\section{Conclusion}

Our xenon EDM experiment based on a novel approach with a sub-millimeter liquid sample and rotating electric fields perpendicular to the Larmor precession axis potentially achieves a statistical sensitivity of $10^{-32} \mathrm{ecm}$ within 150 days of operation. Multiple simultaneous experiments employing different field-configurations allow for a detailed understanding of (new) systematic effects.

Still, by implementing electrodes above and below the liquid ${ }^{129} \mathrm{Xe}$ droplets our EDM setup allows for a "conventional" EDM experiment with a static electric field $E \| B_{0}$ giving similar statistic sensitivity, but different systematic effects.

A realization of this novel experimental approach is in progress at the TU München. This research is supported by the DFG cluster of excellence 'Origin and Structure of the Universe'.

\section{References}

[1] M. Trodden, Rev. Mod. Phys. 71, 1463 (1999)

[2] A.D. Sakharov, JETP Lett. 5, 24 (1967)

[3] C.A. Baker, D.D. Doyle, P. Geltenbort, K. Green, M.G.D. van der Grinten, P.G. Harris, P. Iaydjiev, S.N. Ivanov, D.J.R. May, J.M. Pendlebury et al., Phys. Rev. Lett. 97, 131801 (2006)

[4] M.A. Rosenberry, T.E. Chupp, Phys. Rev. Lett. 86, 22 (2001)

[5] W.C. Griffith, M.D. Swallows, T.H. Loftus, M.V. Romalis, B.R. Heckel, E.N. Fortson, Phys. Rev. Lett. 102, 101601 (2009)

[6] B.C. Regan, E.D. Commins, C.J. Schmidt, D. DeMille, Phys. Rev. Lett. 88, 071805 (2002)

[7] M.V. Romalis, M.P. Ledbetter, Phys. Rev. Lett. 87, 067601 (2001)

[8] T.G. Walker, W. Happer, Rev. Mod. Phys. 69, 629 (1997) 